\title{
Étude socio-terminologique du vocabulaire médical cilubà
}

\author{
Emmanuel Kambaja Musampa, Département de Français-Langues \\ africaines, Institut Supérieur Pédagogique, Mbujimayi, République \\ Démocratique du Congo (emmkamus@yahoo.fr)
}

Résumé: À l'issue d'une enquête (menée à Mbujimayi, en République Démocratique du Congo) auprès du personnel médical et paramédical locuteur et utilisateur du cilubà, langue bantoue, classifiée par Guthrie dans la zone linguistique L31, nous avons récolté, grâce au recours préalable au dépouillement documentaire, 150 termes médicaux français traduits en cilubà. Ces termes ont été soumis à une analyse socio-terminologique double: une analyse morphosyntaxique et une analyse sémantique. Il s'agit d'analyser une terminologie médicale, encore essentiellement véhiculée en français. Cette analyse aboutit à une structure morphosyntaxique et sémantique révèlant que le vocabulaire médical en cilubà est «banalisé», il contient plus de termes simples de la classe 7 et ceux de la classe 12 que les autres. Ces termes sont polysémiques, métaphoriques, métonymiques, synonymiques, ou marqués par la synecdoque et le glissement sémantique. La monosémie n'est pas la règle, contrairement à la vieille tradition en terminologie. Cette étude s'insère dans la terminologie africaine et prépare ainsi à un dictionnaire médical bilingue français-cilubà.

Mots clés: TERMINOLOGIE, VOCABULAIRE BANALISÉ, STRUCTURE MORPHOSYNTAXIQUE, STRUCTURE SÉMANTIQUE, MÉTAPHORE, POLYSÉMIE, SYNONYMIE, MÉTONYMIE, MONOSÉMIE

\begin{abstract}
Socioterminological Study of the Ciluba Medical Vocabulary. At the conclusion of an investigation (led by Mbujimayi, in the Democratic Republic of the Congo) among medical and paramedical staff who are speakers and users of Ciluba, a Bantu language classified by Guthrie in linguistic zone L31, we have collected, by means of recourse to the documentary evidence, 150 French medical terms translated into Ciluba. These terms have been submitted to a twofold socioterminological analysis: a morphosyntactical and a semantic analysis. It deals with analyzing a medical terminology, still basically conveyed in French. This analysis leads to a morphosyntactic and semantic structure which shows that the medical vocabulary in Ciluba has become general, containing more commonplace terms from classes 7 and 12 than from the other classes. These terms are polysemic, metaphoric, metonymic, synonymic or characterized by synecdoche and semantic shift. Contrary to the established tradition in terminology, monosemy is not the rule. This study deals with African terminology, in this way preparing for a bilingual medical dictionary French-Ciluba.
\end{abstract}

Keywords: TERMINOLOGY, GENERAL VOCABULARY, MORPHOSYNTACTIC STRUCTURE, SEMANTIC STRUCTURE, METAPHOR, POLYSEMY, SYNONYMY, METONOMY, MONOSEMY 


\section{Introduction}

La présente étude a été menée dans le cadre d'un vaste projet sur la lexicographie des langues congolaises, et principalement la lexicographie du cilubà, langue bantu parlée au centre de la République Démocratique du Congo et classifiée par Guthrie dans la zone linguistique L31.

Cette étude, première de la série, est une analyse terminologique, vue sous un angle sociolinguistique. Il n'est pas ici question de créer des lexèmes pouvant être utilisés dans le domaine médical; encore que pareille entreprise n'est pas de moindre importance pour une langue non encore dotée de terminologie, dans presque tous les domaines (sauf le domaine religieux)! Seulement, pour l'heure, il nous a semblé important de décrire le vocabulaire médical créé par la communauté linguistique dont nous étudions le parler.

C'est pourquoi nous avons choisi de parler d'étude socio-terminologique. Ceci paraîtrait aux yeux de certains lecteurs un pléonasme vicieux dans la mesure où la terminologie se définit comme «la discipline qui étudie les vocabulaires spécialisés et en analyse les conditions d'utilisation» (http://www. cfwb.be/franca/pg 011.ht). À notre point de vue, il n'est nullement un pléonasme: nous étudions non seulement les conditions d'utilisation mais aussi les mécanismes de création du lexique spécialisé par la communauté.

En effet, il faut standardiser les termes déjà créés par la société, pour permettre la communication et l'assimilation, au lieu de créer d'autres concurrents. La langue est un phénomène social.

De ce qui précède, notre objectif est de décrire sur le plan à la fois morphosyntaxique et sémantique ce vocabulaire «banalisé»1, vocabulaire fort utile parce que servant de trait d'union entre les médecins, le personnel médical ou paramédical et les non-spécialistes (patients ou autres).

Cette description jette les bases d'un lexique médical bilingue françaiscilubà, que nous appelons de tous nos vœux. En outre elle aidera, tant soit peu, le traducteur bilingue (français-cilubà). Nous appliquons la méthode descriptive structurale à l'étude des données lexicales (Bal 1966: 124).

Ce travail n'est certes pas isolé, il s'inspire des lexiques déjà existants, publiés par le Centre d'études de linguistique théorique et appliquée de Kinshasa. Il s'en écarte à deux points de vue: du point de vue de l'objectif et du point de vue de la procédure.

Du point de vue de l'objectif, notre travail n'est pas un travail de vulgarisation du lexique médical, cherchant donc à répondre aux besoins des utilisateurs. Il est un effort de compréhension, par le terminologue-lexicologue de l'objet de son travail. Nous proposerons, à la fin, des options de création terminologique.

$\mathrm{Du}$ point de vue de la procédure, nous ne créons pas de termes, nous n'évaluons pas les équivalences français-cilubà, nous analysons les structures lexicologique et sémantique. 
Notre méthodologie est la suivante: nous avons procédé à une enquête qui consistait à dire en cilubà 150 termes français recouvrant quatre domaines de la médecine, à savoir les pathologies, les actes médicaux, les lieux et les instruments médicaux. Ces lexèmes ont été tirés des ouvrages spécialisés. ${ }^{2}$ Lors de ce dépouillement documentaire, nous avons privilégié les termes les plus courants.

Cette enquête a été menée auprès des locuteurs du cilubà prestant dans le domaine médical: deux infirmiers ayant le grade académique de gradué en Sciences et Techniques médicales, un infirmier technicien A2 (ayant le niveau équivalent à celui d'un D6), un médecin enseignant à l'Université de Mbujimayi.

Comme nous l'avons déjà dit ci-haut, nous utiliserons la méthode descriptive pour analyser nos données. Il s'agit d'une étude qui s'efforce de dégager les structures propres au lexique étudié, sur le plan morphosyntaxique et sur le plan lexico-sémantique.

À l'issue de cette brève introduction, nous proposons au lecteur la discussion des résultats. Il nous a semblé pratique de présenter en annexe les données.

Au cours de la présente étude, nous utiliserons les abréviations et représentations ci-après:

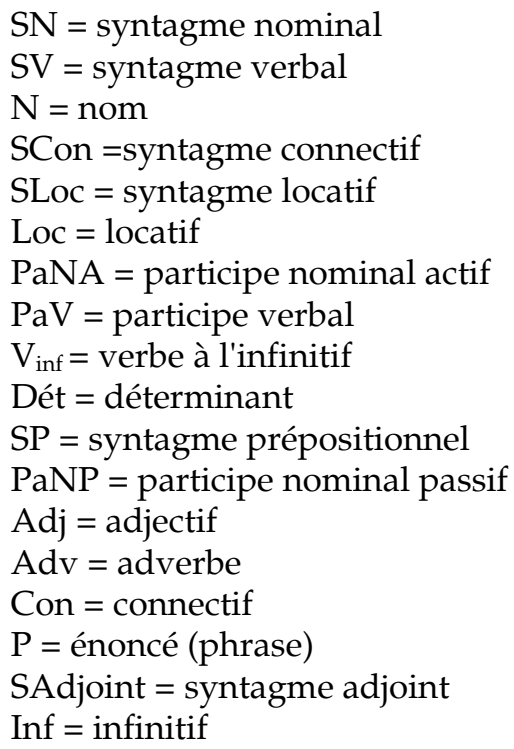

Notez que, dans plusieurs contextes, la voyelle finale du mot lubà devient basse lorsqu'elle est suivie d'une voyelle basse. Dans le cas contraire, elle reste haute. Ceci est une loi phonologique et n'a aucune incidence sur le sens (Exemple: kànsêra aku (ce cancer là) et kansêrà kàdi kàmutàcisha (le cancer le malmène).

$\mathrm{Au}$ regard des résultats d'enquête, notre discussion reposera sur deux questions principales (chacune étant double): 
- Quelle est la structure morphosyntaxique de ce vocabulaire? Quelle application terminologique on peut en faire?

- Quelle est la structure sémantique de ce vocabulaire médical? Quelle implication pour le travail terminologique?

Toutes choses étant égales par ailleurs, notre exposé s'étale sur deux axes:

\section{L'analyse morphosyntaxique}

Le lexique médical en cilubà est largement dominé par les termes de la classe 7. En effet, plus ou moins $21,05 \%$ des termes simples du corpus appartiennent à cette classe qui renvoie aux substantifs désignant les maladies (cilundù, cyuwùjà, cyâdi, ciseki, cikupi, cifù), l'état (cyoyoyi, cyonà), les objets (cipimu, cileledi, cipandidi) ou les résultats d'actions (cisàlu, cipimu)

De ce qui précède, lors de la création des termes, le terminologue doit le plus rechercher dans cette classe, car il y a plus de chances qu'il trouve «la perle recherchée» et que celle-ci vienne à s'adapter bien au système linguistique du cilubà. En procédant ainsi, non seulement il se conforme au génie de la langue, mais aussi il crée des mots que la communauté assimilera vite.

En outre, la classe 12, la classe du diminutif est aussi plus employée que les autres dans la terminologie médicale lubà $(15,79 \%)$. Ceci est plus logique: il s'agit de l'idée de comparaison qui se situe derrière tous ces nouveaux termes. En comparant, par exemple, la particule d'appendice à l'intestin, il est clair que l'on désignera l'appendice par un diminutif en tant que prolongement du caecum.

Certaines classes n'apparaissent pas dans notre corpus: la classe 2 (le pluriel de la classe 1) et la classe de personnes. Cependant ce qui justifie cette absence est le fait que la personne peut aussi être exprimée par la classe 4. Ainsi, l'agglutination des préfixes des classes est compréhensible dans la mesure où les genres $1 / 2+4$ désignent des personnes: on entend également «bamingàngà (les médecins/les infirmiers): le pluriel».

Il est alors très important pour le terminologue de marquer le genre et les autres modifications morphologiques à l'entrée de son article.

Quatorze lexèmes de classe 5 sont des gérondifs, il n'y a dans cette classe qu'un seul substantif (dicì). Ceci est conforme au système du cilubà, dans lequel la plupart des mots de la classe 5 sont des gérondifs (formes nominales du verbe). Nous constatons que le cilubà préfère pour les actes médicaux, les états et les actions, recourir à l'infinitif.

Dans la terminologie médicale lubà, nous trouvons également les emprunts. La plupart d'entre ces néologies sont des calques (il y a aussi des adaptations: bilààdì et lùpitaadì). Tous ces emprunts ont été faits au français, la langue du personnel médical et para-médical.

Le terminologue ne peut s'empêcher de recourir à l'emprunt. D'ailleurs, en le disant ce n'est qu'une pétition des principes. Toutefois il ne doit pas se fier au transcodage du non-spécialiste! 
Quant à l'accord de ces emprunts, il se fait normalement selon que le terme est classé dans l'une ou l'autre classe: àmîbà, àlùkòlà, àpàdìsità, òpitalà, trômpà, kìninà, làbo, kilìnikà ayant la classe ø prennent les préfixes $u$ - et $i$-, c'est-àdire en $1 / 4$. Seuls les termes dont la première syllabe est interprétée comme préfixe de classe s'accordent selon lesdits préfixes: Bìlàà bìdi bìntàcisha (Le bilharziose me tourmente), Dispànsêrà dìdi mutancì mule (Le dispensaire est situé loin d'ici), Kànsêra kàdi kàshebeya bantu bavulè (Le cancer tue beaucoup de gens).

Il n'est pas sans importance de constater que ce vocabulaire emploie une variété de syntagmes nominaux $(\mathrm{SN})$ :

$$
\begin{aligned}
\mathrm{SN} 1= & \mathrm{N}+\mathrm{SN} 2 \\
& \text { Con }+\mathrm{SN} 1 \\
& \mathrm{~N}+\mathrm{SCon} \\
& \mathrm{N}+\mathrm{SLoc} \\
& \mathrm{N}+\text { Adj } \\
& \mathrm{N}+\mathrm{PaNA} \\
& \mathrm{N}+\mathrm{N} \\
& \text { Con }+\mathrm{N}+\mathrm{PaV}
\end{aligned}
$$

Au regard de cette variété, le terminologue dispose d'un éventail de choix pour la création des syntagmes. Les structures que nous avons découvertes sont plus variées pour permettre la création syntagmatique.

Les syntagmes verbaux (SV) ne sont pas eux non plus absents du vocabulaire médical lubà. Dans notre corpus, ils sont aussi en bon nombre (22). Toutefois, par rapport au SV, le SV n'est pas aussi varié. Il n'existe que deux types de $\mathrm{SV}$ : SV à noyau gérondif et SV à noyau infinitif.

Le noyau verbal (gérondif ou infinitif) est suivi d'un complément qui peut être un N, un SN, un SV, un SAdjoint (le circonstanciel).

Notre corpus a aussi révélé le recours à des énoncés pour exprimer les référents médicaux. Certes, ces énoncés ne sont pas nombreux, mais ils présentent une variété au niveau de la base prédicative: tantôt on retrouve une base prédicative, tantôt une base participe nominal, tantôt un participe verbal.

Pour clore cette description morphosyntaxique, nous disons que le vocabulaire médical ici analysé présente plus de formes nominales que de formes verbales et des énoncés. Conséquemment, le terminologue devra privilégier le recours aux formes nominales (principalement les substantifs simples et les syntagmes nominaux).

\section{L'analyse sémantique}

\subsection{La métasémie}

Nous examinerons quelques traits métasémiques de notre vocabulaire: la métaphore, la polysémie, la synecdoque, la métonymie, la synonymie, le glissement morpho-sémantique. 


\section{- La métaphore}

La métaphore est l'une des réalités fondamentales du langage humain. Aucun lexique ne peut être sans métaphore. Car elle n'est pas le fait de la rhétorique, elle est plutôt présente dans toute notre parole. Les terminologues, bilingues ou monolingues, en font quotidiennement l'expérience. À titre de rappel, disons avec Kleiber (1983, cité par Meyer 1988) qu':

il n'y a métaphore que si: (1) le sens littéral ne correspond pas à ce que qu'a voulu dire le locuteur/ou ne renvoie pas à un référent qui fait partie de sa référence virtuelle, (2) la compréhension de ce qu'a voulu dire le locuteur/ou la découverte du référent actuel «étranger» passe par la mise en application de mécanismes de similitude (http://www.info-metaphore.com/definition.html).

Ajoutons avec Dubois (1975: 202) que la métaphore, considérée comme intersection de deux ensembles sémiques, comporte trois unités sémantiques (signifié de départ «D», signifié d'arrivée «A», signifié intersectif «I») dont aucune n'est, en théorie, prééminente par rapport aux autres.

Les termes suivants sont métaphoriques: diboba, kalòwa, kashèèlèlèkà, buzevu, dyuminyina, disaamà dyà diboba, dibùùkà dyà mashi, dijìmina dyà lungènyi, mukàjì kuulu kula, kampala dibàkà, kubàndisha mubidi, kusabula mwâna, kutùùla difù.

Nous avons rangé ces métaphores en quatre catégories:

- les métaphores du devenir,

- les métaphores de la mesure,

- les métaphores de l'espace, et

- la métaphore de la forme.

Notre classification repose sur le principe d'existence d'une catégorie fondamentale, prototypique, autour de laquelle tournent toutes nos métaphores en tant que systèmes de pensée. Nous soutenons le point de vue de Lakoff et Johnson, selon lequel la métaphore est inscrite dans la pensée: «Notre système conceptuel ordinaire, qui nous sert à penser et à agir, est fondamentalement métaphorique» disent-ils (Lakoff et Johnson 1980: 13).

- Les métaphores du devenir

Sous cette catégorie, nous rangeons les métaphores suivantes: (1) diboba (kwashiorkor); (2) dyuminyina (convulsion).

Le changement d'état est une réalité vitale. Pour l'exprimer, le vocabulaire médical, que nous analysons, recourt à la métaphore du devenir. L'énoncé (1) renvoie à un passage $\mathrm{du} \mathrm{cru}, \mathrm{du}$ fondamentalement premier, de la couleur première à ce qui a perdu son premier état sous l'effet d'un quelconque agent. Une personne en bonne santé, un objet en bon état sont comparés à un fruit cru. L'on entend dans le langage courant: màshinyì mabìshi, mutèèlù mubìshi, nsongàkàjì ùcìdi mubìshi. Littéralement on traduira: véhicule cru (pour dire un véhicule neuf), une chemise crue (pour dire une chemise neuve), une fille encore crue 
(pour dire une fille vierge).

Diboba se dit pour une personne qui présente les signes évidents de malnutrition. La pensée littérale est celle-ci: le corps de ce malade est passé (devient) de sa couleur première, la couleur de la vitalité, de la rudesse comme le cru, à la couleur d'un objet cuit, sous l'effet de la chaleur (ou du feu).

Une autre métaphore du devenir est la (2). Il est ici question du passage de ce qui était pliable à ce qui est devenu non-flexible, dur au toucher et allongé, sans vie, donc. L'énoncé mwâna ìmuumìnyìna se traduit littéralement par *l'enfant est devenu non-flexible. Il ressemble ainsi à du bois sec, un bois sans vie.

Comme on le voit, les deux métaphores du devenir s'inspirent des réalités d'autres domaines pour exprimer celles qui sont du domaine médical. Le passage du cru au mûr relève du domaine des objets (surtout d'aliments) et le passage du flexible (de l'humide ou du mou) vers le non-flexible (le dur) relève du domaine de la matière.

\section{- Les métaphores de la mesure}

Buzevu est l'une des illustrations de la métaphore in absentia attestée dans le vocabulaire médical sous analyse. Elle est, heureusement, une métaphore de la mesure. En effet, ce terme renvoie à l'éléphantiasis qui est augmentation considérable du volume d'un membre ou d'une partie du corps due à l'obstruction des vaisseaux lymphatiques. En régions intertropicales, la cause majeure d'éléphantiasis est la filariose lymphatique, transmise par les moustiques (voir http://www.chu-rouen.fr/ssf/pathol/elephantiasis.html).

Dans le parler lubà, ce gonflement est comparé à la taille de l'éléphant (nzevu). Morphosémantiquement, le terme pourrait être analysé en bu- (le morphème de classe 14) et -zevu (le thème nominal). C'est une heureuse équivalence entre la désignation et le référent, étant donné qu'il y a ici une motivation linguistique.

Le terme kashèelèlèkà renvoie d'abord à une pathologie qui se manifeste par le blocage de la croissance de l'enfant, accompagné des amaigrissements, la perte d'appétit, les pleurs sans arrêt. Cette pathologie est souvent associée à un mauvais sort. Jusqu'à ce jour, elle se traite mieux chez des femmes supposées détentrices du pouvoir de jeter et de lever ce sort. La pauvre victime de ce maléfice maigrit au point de rester la peau collée aux os, les veines amplement visibles, on dirait des filaments.

Ce terme renvoie à la réduction de la mesure, mieux à la réduction de la taille. On trouve dans le langage courant un autre adjectif du même radical shèè - kashè̀pètèka/mushèpèteka (pour dire devenu de très courte taille; lorsqu'on parle d'une personne, d'un animal). Ainsi donc, nous comprenons pourquoi ce terme est aussi utilisé pour désigner le sida. Un adulte ou un enfant atteint du sida (kashèèlèlèkà) ne cesse de maigrir.

- $\quad$ Les métaphores de l'espace

Le vocabulaire médical exprime certains référents au moyen des métaphores de l'espace ci - après (retrouvées dans notre corpus): (1) kuulu kula (la grossesse: 
littéralement «être suspendu plus haut»), (2) kusabula mwâna (faire accoucher par césarienne: littéralement «sortir d'un trou plein d'eau un enfant»), (3) dibùùkà dyà mashi (l'anémie: littéralement «l'envol du sang»), (4) kubàndisha mubidi (faire la fièvre: littéralement «hausser le corps»).

La métaphore (1) renvoie à l'espace - hauteur. La femme qui est grosse est comparée à une personne située à une très haute altitude. Ceci suffit pour comprendre toute la peur qui entoure l'accouchement. (Disons au passage qu'on entend toujours dire les femmes: kudilela sè ǹku lufù; littéralement traduit par: qui va à l'accouchement s'expose à la mort. L'accouchement est un danger capable de donner la mort. La femme qui attend famille se sent toujours en «insécurité», car le haut est insécurisant, tandis que le bas est rassurant. Lorsque la femme s'accouche, on entend dire: mukàjì wàátùùluki bilenga; littéralement: la femme est bien descendue. La métaphore de la hauteur est très forte: le haut n'est pas rassurant, le bas l'est au contraire. Le haut est synonyme de danger de mort permanent.

La métaphore (2) renvoie à l'espace - trou. Son équivalent français césarienne est une métonymie. Kusabula mwâna désigne littéralement: retirer l'enfant d'un trou dans lequel on trouve également de l'eau. Cette métaphore renvoie à l'idée que l'enfant baigne dans une cavité (ici l'utérus) dans laquelle il y a de l'eau (le liquide amniotique). Un autre sème attaché au terme kusabula mwâna est celui de «danger». L'enfant que l'on tire «par césarienne» est supposé avoir été en danger de mort, car ne pouvant être expulsé normalement.

La métaphore (3) renvoie à l'espace - étendu, à travers lequel l'intelligence, ici considérée comme un objet, est égarée. Kujìmija lungènyi (littéralement: égarer l'intelligence) s'oppose à kupeta lungènyi (re)trouver l'intelligence, littéralement: pour dire retrouver l'exercice de sa faculté mentale). L'on considère que celui qui développe des troubles «égare ses facultés mentales» ou même que celles-ci sortent de lui. D'où une métaphore que l'on entend couramment kupingaja lungènyi (littéralement: retourner l'intelligence, à sa place).

La métaphore (4) est aussi une métaphore de l'espace - hauteur, comme la (1). Dibùùkà dyà mashi est traduit littéralement par: l'évaporation du sang. Le sang s'évapore vers le haut. Là où le français exprime la privation (an-), le cilubà exprime la fuite, la perte. Le terme dibùùkà dyà mashi désigne l'anémie, due à une fièvre (souvent). Lorsque l'anémie est due à une autre cause, on parle de dijika dyà mashi (littéralement traduit par: l'épuisement du sang). Il n'est pas faux de dire que le sang est considéré comme l'eau (par son trait «liquide») chauffée par un pathogène à une température telle que l'évaporation est possible. C'est pourquoi, la fièvre est désignée par la température (mubidi kapyà, mubidi mupyà); littéralement l'on traduirait par: le corps est chaud.

\section{- La polysémie}

Le terme "polysémie» se définit toujours par opposition à la monosémie et à l'homonymie. Nous ne voudrons pas, pour l'heure en tout cas, nous replonger dans le vieux débat sur ces aspects des problèmes sémantiques. Nous pensons 
que le lecteur intéressé, pourrait lire les ouvrages de Kleiber (1999) et Pergnier (1993) cités à la bibliographie de la présente étude.

Somme toute, nous définissons la polysémie comme le fait qu'un mot revêt une pluralité des sens, lesquels sont unis par un certain rapport. Pareille définition nous permet de nous éloigner de l'homonymie et des prototypes. Elle nous permet également d'éliminer de notre champ d'étude, les mots vagues.

Avant d'entrer dans le vif de l'analyse, disons qu'il est un fantasme dont nous devons nous défaire: celui de la monosémie de la terminologie scientifique. Notre corpus prouve (je pense que d'autres, même les plus hautement construits, le prouveront encore) que les termes peuvent aussi être polysémiques.

La polysémie tient de la nature même du signe linguistique. Le terminologue doit cesser de ramener la signification au sens et savoir que le terme peut désigner plusieurs référents selon les «contextes».

Venons-en aux polysèmes du corpus: (1) mâyi, (2) diteeta/mateeta, (3) musàndà, (4) cipimu, (5) difù, (6) mungàngà, (7) mubidi.

- $\quad$ mâyi: Seul le contexte en précise le sens.

Dans l'énoncé (1) Bàdi bàmwela mâyi bwalu ùdi nè màlàrìya (Il est sous perfusion parce qu'il fait de la malaria), mâyi renvoie au soluté de perfusion.

Dans un autre énoncé du genre (2) Mungàngà ùdi ùkòka mâyi àà mu mwongu (L'infirmier fait une ponction lombaire), le terme mâyi désigne le liquide céphalo-rachidien.

Dans un troisième énoncé (3) Mwâna ewu m̀munwà mâyi pa kulediibwa (Le bébé a inhalé le liquide amniotique à la naissance), le terme mâyi désigne le liquide amniotique.

Il signifie le sperme dans le contexte suivant: (4) Bàyendà kêna nè bilùmà mu mâyi èndà to (Le sperme de son mari manque des spermatozoïdes, c'est-à-dire ce qui s'appelle azoospermie).

On entend lors des séances d'éducation sanitaire, au sujet du traitement de la diarrhée, l'infirmier dire (5) Pàdì mwâna wèla munda, bìdi bikèngela kumunwìsha mâyi misangu mivulè (Quand un enfant fait de la diarrhée, il faut lui donner régulièrement de l'eau à boire, c'est-à-dire pour prévenir la déshydratation).

Enfin, le terme mâyi dans le dernier contexte, (6) ùdi nè mâyi ku bisùlusulu (Il a du liquide dans la cavité pleurale), désigne le liquide qui emplit la cavité pleurale.

Comme on le voit, le terme polysémique mâyi ne peut nullement être confondu: le contexte précise toujours le sens. Patients et personnel de santé communiquent sans aucun heurt.

- diteeta/mateeta: Ce terme a plus d'une désignation. Dans l'énoncé (1) Ndi mufila bunyaù bwà mungàngà èenzà mateeta (J'ai donné les selles au laboratoire pour examens), il désigne les analyses des échantillons au laboratoire.

Dans un autre énoncé du type: (2) Ngaayi nè mwâna kwà mungàngà, wèénjì mateeta mubidi mujimà, kêna musangàna cintu (Le médecin a ausculté tout le 
corps de l'enfant que je lui ai amené, mais hélas, il n'a rien trouvé!), le terme désigne l'auscultation. Dans un cas comme dans l'autre, le terme désigne le fait d'examiner un échantillon ou un malade.

_ $\quad$ musàndà: Ce terme est également polysémique. Nous pouvons le voir à travers ces énoncés:

(1) Mpùnga udi nè misàndà; ke bwalu kaayì ùdi nè difù dipàta (Mpùnga a des verminoses, raison pour laquelle il présente un ballonnement de ventre).

(2) Mpùnga ùdi ùnàya ku makàsà kutupù mu moùla, neàdyatà misàndà (Par le fait qu'il joue pieds nus sous la pluie, Mpùnga aura à marcher sur des vers de terre).

Dans les deux énoncés, le terme misàndà (plur. de musàndà) n'a pas le même sens: il signifie tantôt «verminose» tantôt «vers de terre». C'est pareil aussi pour le français.

- cipimu: Ce terme désigne tantôt la pesée, tantôt la consultation prénatale, tantôt le prélèvement de la température. C'est cela que nous comprenons à travers les énoncés suivants:

(1) Ndààku nè mwâna ku cipimu (Amène l'enfant à la pesée).

(2) Pàdì mukàjì nè difù, bìdi bikèngela ì̀kalà ùya ku cipimu (Il est recommandé à la femme qui attend famille d'aller régulièrement à la consultation prénatale).

(3) Cipimu cyà mwâna cìvwa àmu mu makùmi ànaayi (La température de l'enfant était stationnée à $40^{\circ} \mathrm{C}$ ).

- difù: Il désigne tantôt la grossesse, tantôt le ventre, tantôt les coliques. Soient les énoncés:

(1) Kapinga ùdi nè difù (Kapinga est grosse).

(2) Mungàngà ùdi ùbaabata mu difù dyà mubèè̀ (Le médecin tâte le ventre du malade).

(3) Difù dìdi dìmunyènga (Il a des coliques abdominales). Cet énoncé est l'équivalent de munda mùdi mùmunyènga qui ce traduit littéralement par: Ce qui est à l'intérieur du ventre me torsionne.

Comme on le constate à travers ces énoncés, le contexte discursif et phrastique lève toute possibilité éventuelle de confusion.

- mungàngà: Ce terme désigne tantôt docteur/médecin, tantôt l'infirmier, tantôt le laborantin, tantôt le tradi-praticien. Nous le voyons dans les énoncés ci-après:

(1) Mungàngà wàátèèki mwâna panshì (L'infirmier a circoncis l'enfant). Mungàngà a le sens d'infirmier.

(2) Dans Mungàngà wàángàcì mashi àà mu munu (Le laborantin a fait la goutte épaisse), le terme désigne le laborantin. En parlant à un médecin, le terme a le sens de médecin ou de docteur: Mungàngà wânyi, ndi nè cyâdi kùdi ngondu isambòmbò (Cher docteur/médecin, voici six mois que j'ai la tuberculose). Le terme est aussi utilisé pour désigner le pharmacien. Enfin, mungàngà est employé pour quiconque soigne. C'est pourquoi il s'emploie pour le tradi- 
praticien aussi (celui qui soigne à base de plantes comme dans la tradition; sans avoir été dans une école de médecine). lui-même.

Le terme mubidi désigne tantôt la fièvre, tantôt la santé, tantôt le corps

Dans l'énoncé (1) Mwâna ùdi ùsaama mubidi, le terme désigne la fièvre. Cet énoncé se traduit par: L'enfant fait la fièvre. Mais admettons qu'on se trouve à une séance de vaccination, l'infirmier pourrait dire à une maman: (2) Mwanèèbà kêna nè mubidi mwîmpà to. Dans ce contexte, le terme désigne l'état de santé. Cet énoncé se traduirait par: Ton enfant n'a pas bonne santé.

L'énoncé (3) Mbîmpà kulama mubidi wèbà nè mankendà bwà kwepuka twîshi, dit par une infirmière à une maman lors de la consultation prénatale, contient le terme mubidi qui désigne le corps. Il se traduirait par: Il est mieux de garder ton corps propre en vue d'éviter des infections (en cette période de grossesse).

Enfin, par euphémisme, le terme mubidi est employé pour désigner le sexe ou les rapports sexuels: (4) Mwanèè̀ wa balùma eu, ùkaadi mumanyà mubidi wà muntu mukàjì pàdìye apa (Ton fils que voici a déjà eu des rapports sexuels avec une femme).

Comme on peut le constater, la polysémie lexicale est un fait attesté dans le lexique médical. Une note cependant est à faire: la polysémie n'affecte pas les syntagmes nominaux (ou verbaux). Elle est limitée aux mots dits simples: la longueur de l'unité lexicale lève l'ambiguïté et la polysémie.

Dans le même ordre d'idées, nous analyserons également le phénomène de synecdoque.

\section{- La synecdoque et la métonymie}

Nous étudions les deux métasémies ensemble parce que la première n'est qu'un cas particulier de la deuxième. Pour rappel, la métonymie consiste à nommer un concept au moyen d'un terme désignant un autre concept, lequel entretient avec le premier une relation d'équivalence ou de contiguité. Et la synecdoque est une métonymie particulière qui consiste à considérer tout pour la partie, la partie pour le tout, un élément pour l'ensemble.

En effet, synecdoque et métonymie ne sont pas à rechercher dans le vocabulaire médical lubà. Les termes mucìma, dicì, cifù, cyâdi, munda, et mutù sont des synecdoques. Difù et mubidi sont aussi métonymiques.

Certaines maladies sont exprimées en termes de parties où elles se localisent. Ainsi trouve-t-on mutù (littéralement: tête) pour désigner les céphalées ou les migraines, mucìma (littéralement: le foie) pour désigner l'hépatite $\mathrm{A}, \mathrm{B}, \mathrm{C}$ ou la cirrhose de foie, dicì (littéralement: oreille) pour désigner toutes les maladies de l'oreille, cifù (littéralement: estomac) pour désigner la gastrite, cyâdi (littéralement: la poitrine) pour désigner la tuberculose, munda (littéralement: l'intérieur) pour désigner la diarrhée ou autres pathologies internes. Les termes difù et mubidi sont également métonymiques. Difù qui se traduit littéralement par ventre peut, dans un autre contexte, comme le suivant, désigner la grossesse: 
(1) Kapinga ùdi nè difù (Kapinga est grosse). C'est la métonymie du contenant pour le contenu. En effet, toute personne, mâle ou femelle, a un ventre (difù). Cependant, dans le cas d'espèce, il s'agit de l'enfant logé dans l'utérus, lequel est placé dans le petit bassin (ou pelvis) féminin. Au cours de la croissance de l'embryon, puis du fœtus, le volume du ventre augmente. Alors, le locuteur du cilubà parle du ventre (le contenant) au lieu du fœtus, le contenu.

Enfin le terme mubidi est aussi à la fois métaphorique et polysémique. Dans l'énoncé Mwâna ùdi ùsaama mubidi (L'enfant fait de la fièvre), le terme mubidi désigne la température du corps. Il est dans ce cas métonymique.

\section{- La synonymie}

C'est bien étrange de parler de synonymie dans un vocabulaire médical (une terminologie). Cependant, il ne serait pas honnête de notre part de ne pas en parler, car elle existe. Au cours de notre analyse du corpus, un seul cas de la synonymie s'est révélé.

Disons de prime abord que la synonymie se classe selon quatre types (d'après Kerbrat-Orecchioni 1979: 168-169):

- la synonymie complète et totale (extrêmement rare),

- la synonymie complète non-totale, e.g. beau-fils/gendre,

- la synonymie totale non-complète (extrêmement fréquente, la connotation qui oppose les deux synonymes pouvant être de nature extrêmement diverse), e.g. jaunisse/ictère (terme commun/terme technique); soixante/+ septante (connotation géographique); cacher/celer (connotation archaïque), et

- la synonymie non-totale ni complète: la plus fréquente, e.g. fermer/clore.

Il ressort de cette classification que le seul cas de synonymie attestée dans notre corpus est double. D'abord, il est celui d'une synonymie complète et totale: elle est attestée pour le triplé mukupa/dibùluka/dipaala. C'est cette synonymie que définit Ullmann quand il dit: «on ne peut appeler synonymie que les mots qui peuvent se substituer l'un à l'autre dans un contexte sans le moindre changement dans la portée cognitive ou affective» (cité par Kerbrat-Orecchioni 1979: 167).

On peut dire, en interchangeant les trois premiers termes que nous avons le même énoncé:

(1) Ilunga ùdi nè dipaala, ke bwalu kaayì ùdi ùdya byà ku diyaala.

(2) Ilunga ùdi nè dibùluka, ke bwalu kaayì ùdi ùdya byà ku diyaala.

(3) Ilunga ùdi nè mukupa, ke bwalu kaayì ùdi ùdya byà ku diyaala.

Ces trois énoncés se traduisent par un seul «équivalent»: Ilunga est devenu fou, $c^{\prime}$ est pourquoi il mange aux ordures.

Ensuite, katombùtombù, le dernier terme est, par rapport aux trois précédents, en synonymie totale mais non-complète. Car la folie dont il est question 
pour katombùtombù est une folie intermittente. Ce qui explique qu'il se trouve des contextes dans lesquels ce terme ne peut être interchangeable avec les trois autres.

\section{- Le glissement morpho-sémantique}

Nous désignons ainsi le sens qui se manifeste lors du passage du singulier vers le pluriel dans le cas des termes: kîshi, twîshi, dìsaamà dyà mashi et màsaamà àà mashi.

Les termes kîshi et twîshi ne sont pas identiques sémantiquement, dans certains contextes. Nous en voudrons pour illustrations les énoncés:

(1) Bàyeebà ùdi nè kîshi (Ton mari est sidéen/séropositif/tuberculeux).

(2) Bàyeebà ùdi nè twîshi mu mênyi (Ton mari a des microbes dans les urines).

(3) Mbangùla kîshi ku mênyi (On a découvert, à l'issue de l'examen d'urine, que la personne était séropositive).

(4) Mbangùla twîshi ku mênyi (Les microbes ont été découverts après examen d'urine).

Dans le premier énoncé, le terme kîshi peut désigner le sida, ou la tuberculose (les deux peuvent apparaître en association). Ce qui ici le sème commun est le caractère «très contagieux». Donc, dans ce contexte, kîshi n'est pas le singulier de twîshi. Il ne peut accepter, d'ailleurs, le pluriel. Cet énoncé se traduirait par: Ton mari est séropositif (ou tuberculeux).

Dans le deuxième énoncé, twîshi désigne les germes pathogènes en général, les microbes. L'énoncé (2), en cas d'une infection urinaire, se traduirait par «ton mari a des infections urinaires». De ce qui précède, (3) se traduirait: «on a découvert le sida lors de l'analyse des urines» et (4) «on a découvert les microbes responsables d'infections urinaires lors des analyses».

Dans le même ordre d'idées, dìsaamà dyà mashi ne peut être considéré, dans tous les cas comme le singulier de màsaamà àà mashi. Prenons les énoncés ciaprès:

(5) Mwâna m̀mmufwà dìsaamà dyà mashi.

(6) Màsaamà àa mashi àdi àshipa bâna bikolà.

L'énoncé (5) se traduirait par: «L'enfant est décédé d'une drépanocytose» alors que le (6) se traduirait par: «Les maladies liées au sang tuent beaucoup d'enfants». Si dans l'énoncé (5) le singulier est précis, dans l'énoncé (6), le pluriel renvoie au général, de la leucémie à la drépanocytose.

\subsection{La monosémie}

Nous avons choisi de parler de la monosémie en passant parce que, du point de vue sociolinguistique, elle est une exception qui n'infirme pas la règle selon laquelle les lexèmes sont principalement polysémiques. 
Certes, dans notre lexique, il n'a pas manqué des lexèmes monosémiques. Ils sont aussi nombreux (tous ceux qui n'ont pas fait l'objet de la métasémie). Il nous semble que la raison de la monosémie est la recherche de la rigueur dans la terminologie, caractéristique essentielle pour toute discipline scientifique.

\subsection{Propositions de traduction terminologique}

À l'issue de l'analyse ci-dessus, il nous revient de proposer quelques traductions, sans aucune prétention à la perfection. Ces propositions sont suivies, chacune de petites explications socioterminologiques. Mais il n'est pas ici temps de nous étaler sur les différentes techniques traductologiques utilisées. Nous avons retenu 18 termes:

(1) méningite: cisèbasebabongù $7 / 8$

Ce terme est une lexie composée qui peut s'analyser en cisèba qui désigne le terme méninge. Ce terme est déjà connu dans la langue lubà dans laquelle il désigne peau. Dans ce contexte il renvoie à la membrane qui recouvre le cerveau, désigné par le terme courant bongù. Il y a ici une duplication du terme cisèba pour signifier l'inflammation de la membrane (littéralement: la membrane du cerveau qui grossit). Nous avons mis ensemble les lexèmes: cisèbaseba cyà bongù. Le connectif a été supprimé.

Dans le même ordre d'idées les termes de la même famille se diraient, pour le méningisme: busèbasebabongù 14/0; méningiose se traduirait par sebabongùnènè $0 / 4$ qui signifie en général toute inflammation de méninges.

(2) pédiatrie: bungàngâna 14/0 qui s'analyserait en bungàngà (la médecine) bwà bâna (des enfants) avec le thème nominal -âna (signifiant enfant). De la sorte le terme chirurgie se traduirait par bungàngàpandi 14/0 (la médecine d'opération). La gynécologie serait bungàngànkàji 14/0 (la médecine des femmes) et la médecine interne se traduirait par bungàngàmùndà 14/0 (la médecine de l'intérieur).

(3) globule: kabùlùngù 12/13. Ce terme existe déjà en cilubà et désigne un petit objet rond. Il nous semble approprié pour désigner «globule». Dans cet ordre d'idées, le terme "globule rouge» sera désigné par kabùlùngù mashi. Ce composé désigne directement le sang car le globule rouge est la cellule de sang qui contient l'hémoglobine désigné par métonymie, en cilubà, par le terme mashi (le sang).

(4) hyperglycémie: bòdyòdyòsùkâdì $0 / 4$. En effet, en cilubà, le verbe kubòdyodyoka désigne proliférer, être en grande quantité. C'est bien là l'idée d'une hyperglycémie, terme qui renvoie à l'excès de glucose dans l'organisme. Il est aussi un composé réunissant bòdyodyoka (le verbe) et le substantif sùkâdì (sucre).

(5) hypoglycémie: De ce qui précède, il y a lieu de créer le terme shèèpèsùkâdì $0 / 4$ composé de la racine -shèèpe- qui désigne le fait de se réduire, de diminuer de taille, de devenir petit. Ce qui arrive effectivement dans le cas d'une hypoglycémie. 
(6) hypocalcémie: shèèpèkàlìsyumù $0 / 4$ dans lequel nous retrouvons la racine -shèèpe- dont nous venons de parler et kàlìsyumù 12/13 est le terme courant utilisé par les locuteurs du cilubà, terme emprunté au français pour désigner le calcium. De la sorte la calcémie se traduirait par bùlìsyumù 14/4.

(7) leucémie: se traduirait par mbùdikabùlùngù $1 / 4$, lexème composé à partir kubùdika (se multiplier, devenir en surnombre) et bùlùngù de ka/tu - bùlùngù (terme par lequel nous avons traduit «globule». La leucémie est ainsi entendue comme une prolifération des globules blancs ou autres dans le sang.

(8) virus: kîshincii 12/13 lexème composé de kîshi (petit insecte) et l'adjectif ncii qui signifie «très petit». Dans cet ordre d'idées, le virus est défini comme un petit (très petit alors) organisme qui demeure dans la cellule.

(9) hémoglobine: mpùlùnkùnzù $1 / 4$. Ce terme est un lexème composé de mpùlù qui désigne un petit fruit rouge dit en cilubà mpùlùmànjè et kùnzù qui est adjectif de couleur désignant la couleur rouge. Pour dire que l'hémoglobine est une protéine qui donne sa couleur rouge au sang.

(10) anémie: shèmashi $0 / 4$. Ce terme est lié à ceux que nous avons déjà créés plus haut sur base de la composition verbo-adjectivale -shèè-: diminuer, s'épuiser, et mashi qui désigne dans le langage courant «le sang».

(11) antibiotique: mulùngwìshì $3 / 4$, un terme composé de deux substantifs, à savoir mulùngù (poison) et -îshi (insecte dont nous avons déjà parlé (voir virus).

\section{Conclusion}

À quelle conclusion nous a conduit cette étude socioterminologique?

Nous sommes arrivé à des résultats tels que le vocabulaire médical de la communauté linguistique lubà, dans son état actuel, présente la structure sémantique et même morpho-syntaxique de la langue courante. De ce fait, c'est un vocabulaire banalisé.

Sur le plan terminologique, il est conséquent que le terminologue se débarrasse des vieux fantasmes qui l'empêchaient de voir les métasémies qui jalonnent la terminologie. La métaphore, par exemple, est inscrite, non dans la langue, mais dans notre système de pensée.

Certains traits sont fréquents, d'autres non. Au terminologue de s'inspirer de cette répartition morpho-syntaxique et lexico-sémantique pour son travail sur la terminologie médicale en lubà élaborée systématiquement.

Nous avons nous-même proposé une terminologie constituée de 19 termes en vue de montrer les voies à suivre en vue d'un travail lexico-terminologique utile. Notre espoir est que plusieurs terminologues du domaine lubà s'engageront dans cette voie. 


\section{Notes}

1. Par vocabulaire banalisé, nous entendons le vocabulaire accessible à une population plus ou moins nombreuse, mais nécessairement plus nombreuse que celle qui possède la compétence du langage spécialisé dont il est issu, et moins nombreuse que celle qui ne connaît que le langage courant (Galisson 1979: 125)

2. Il s'agit de Bernard et Pierre (1989), Janssens et Courtejoie (1996) et Manuel du centre de santé (1988).

\section{Bibliographie}

Bal, W. 1966. Introduction aux études de linguistique romane — avec considération spéciale de la linguistique française. Paris: Didier.

Bernard, G. et G. Pierre. 1989. Dictionnaire médical pour les régions tropicales. Kangu-Mayumbe: BERPS.

CHU Hôpitaux de Rouen. 18 février 2006. Le Catalogage et l'Indexation des Sites Médicaux Francophones (CISMeF): éléphantiasis. http://www.chu-rouen.fr/ssf/pathol/elephantiasis.html [17 mai 2006].

Detienne, C. 23 février 2006. La métaphore en question. http://www.info-metaphore.com/ definition.html [17 mai 2006].

Galisson, R. 1979. Lexicologie et enseignement des langues. Paris: Hachette.

Guthrie, M. 1948. The Classification of Bantu Languages. Londres: Oxford University Press.

Janssens, P. et J. Courtejoie. 1996. Aide-mémoire pour le dispensaire. Kangu-Mayumbe: BERPS.

Kerbrat-Orecchioni, C. 1980. L'énonciation: de la subjectivité dans le langage. Paris: Armand Colin.

Kleiber, G. 1999. Problèmes de sémantique - La polysémie en question. Paris: Presses universitaires du Septentrion.

Lakoff, G. et N. Johnson. 1980. Les métaphores dans la vie quotidiennes. Paris: Éd. De Minuit.

Manuel du centre de santé. Vol. I: Soins curatifs. 1988. Kinshasa: Projet de santé pour tous.

Pergnier, M. 1993. Les fondements socio-linguistiques de la traduction. Édition remaniée. Lille: Presses universitaires de Lille. 


\section{ANNEXE 1: Classement des termes}

\section{Les formes nominales}

\subsection{Les substantifs}

\subsubsection{Selon les classes}

\begin{tabular}{|c|c|c|c|c|c|c|c|c|c|c|c|c|c|c|c|c|}
\hline $\begin{array}{l}\mathrm{Cl} 1 \\
\mathrm{mu}-\end{array}$ & $\begin{array}{l}\mathrm{Cl} 2 \\
\text { ba- }\end{array}$ & $\begin{array}{l}\mathrm{Cl} 3 \\
\mathrm{mu}-\end{array}$ & $\begin{array}{l}\mathrm{Cl} 4 \\
\text { mi- }\end{array}$ & $\begin{array}{c}\mathrm{Cl} 5 \\
\text { di- } \\
\end{array}$ & $\begin{array}{l}\mathrm{Cl} 6 \\
\text { ma- }\end{array}$ & $\begin{array}{l}\mathrm{Cl} 7 \\
\text { ci- } \\
\end{array}$ & $\begin{array}{c}\mathrm{Cl} 8 \\
\text { bi- } \\
\end{array}$ & $\begin{array}{c}\mathrm{Cl} 11 \\
\mathrm{lu}- \\
\end{array}$ & $\begin{array}{c}\mathrm{Cl} 12 \\
\text { ka- }\end{array}$ & $\begin{array}{c}\mathrm{Cl} 13 \\
\text { tu- }\end{array}$ & $\begin{array}{c}\mathrm{Cl} 14 \\
\text { bu- }\end{array}$ & $\begin{array}{c}\mathrm{Cl} 15 \\
\mathrm{ku}-\end{array}$ & $\begin{array}{c}\mathrm{Cl} 16 \\
\text { pa- }\end{array}$ & $\begin{array}{c}\mathrm{Cl} 17 \\
\mathrm{ku}-\end{array}$ & $\begin{array}{c}\mathrm{Cl} 18 \\
\mathrm{mu}-\end{array}$ & Tot. \\
\hline 5 & 0 & 6 & 4 & 4 & 3 & 12 & 2 & 6 & 9 & 2 & 3 & 0 & 0 & 0 & 1 & 57 \\
\hline
\end{tabular}

\subsubsection{Selon le genre}

\begin{tabular}{|l|l|l|l|l|l|l|l|}
\hline $1 / 0$ & $1 / 4$ & $0 / 4$ & $5 / 6$ & $5 / 0$ & $0 / 6$ & $7 / 8$ & $0 / 8$ \\
\hline $\begin{array}{l}\text { mfwènka } \\
\text { nkooyi } \\
\text { mpalù } \\
\text { nsàdì } \\
\text { mpùta }\end{array}$ & $\begin{array}{l}\text { mungàngà } \\
\text { (ngàngàbukà) } \\
\text { mutù } \\
\text { munànà } \\
\text { mukupa } \\
\text { mucìma } \\
\text { musàndà }\end{array}$ & $\begin{array}{l}\text { milubàlubà } \\
\text { mpùsù } \\
\text { misònji } \\
\text { mbàlàngà }\end{array}$ & $\begin{array}{l}\text { diteeta } \\
\text { dicì }\end{array}$ & $\begin{array}{l}\text { dibùluka } \\
\text { dipaala }\end{array}$ & $\begin{array}{l}\text { màsàsà } \\
\text { mâyi } \\
\text { mankùbùngu }\end{array}$ & $\begin{array}{l}\text { cilundù } \\
\text { cyoyoyi } \\
\text { cyonà } \\
\text { cipimu } \\
\text { ciseki } \\
\text { cikupi } \\
\text { cifù } \\
\text { cileledi } \\
\text { cipandìma } \\
\text { cyâdi } \\
\text { cyuwàjà } \\
\text { cisàlu }\end{array}$ & \\
\hline 5 & 6 & 4 & 2 & 2 & 3 & 12 & 2 \\
\hline
\end{tabular}

\begin{tabular}{|c|l|l|l|l|l|l|l|}
\hline $11 / 4$ & $11 / 0$ & $12 / 13$ & $0 / 13$ & $14 / 0$ & $14 / 6$ & $18 / 0$ & Total \\
\hline $\begin{array}{l}\text { lusòngà } \\
\text { lusundu } \\
\text { lukosù } \\
\text { lushìngì }\end{array}$ & $\begin{array}{l}\text { lùbùngù } \\
\text { londàpu }\end{array}$ & $\begin{array}{l}\text { kalwalwalwa } \\
\text { kîshi } \\
\text { kakèla } \\
\text { katombùtombù } \\
\text { kalòwa } \\
\text { kàntembela } \\
\text { kambulu } \\
\text { kashèèlèlèkà } \\
\text { kasèndà }\end{array}$ & $\begin{array}{l}\text { twîshi (selon } \\
\text { le contexte) } \\
\text { tunèka }\end{array}$ & $\begin{array}{l}\text { buzevu } \\
\text { bupofu }\end{array}$ & bulààlu & munda & \\
\hline 4 & 2 & 9 & 2 & 2 & 1 & 1 & 57 \\
\hline
\end{tabular}




\subsection{Les gérondifs}

Nous avons recensé 11 gérondifs appartenant au genre 5/0:

$$
\begin{array}{ll}
\text { - } & \text { diboba } \\
\text { - } & \text { dikùluka } \\
\text { - } & \text { dilènga } \\
\text { - } & \text { dilèwuluka } \\
\text { - } & \text { dipaluka } \\
\text { - } & \text { dipanda } \\
\text { - } & \text { ditàpika } \\
\text { - } & \text { diteeta } \\
\text { - } & \text { dyondapa } \\
\text { - } & \text { dyuminyina } \\
\text { - } & \text { dyumusha }
\end{array}
$$

\subsection{Les emprunts}

Il s'agit de termes que l'on reconnaît encore comme étrangers, car il faut reconnaître que beaucoup de mots étrangers entrés dans le lexique lubà fonctionnent tellement bien que même certains locuteurs natifs ont du mal à les reconnaître comme tels. Les 10 emprunts que nous avons retrouvés dans notre lexique sont:

$$
\begin{array}{ll}
\text { - } & \text { àlùkòlà } \\
\text { - } & \text { àpàndìsità } \\
\text { - } & \text { bìlààì } \\
\text { - } & \text { dìspansêrà } \\
\text { - } & \text { kànsêrà / kànsêlà } \\
\text { - } & \text { kìlìnikà } \\
\text { - } & \text { làbo } \\
\text { - } & \text { kìninà } \\
\text { - } & \text { òpìtalà / lùpìtaadì } \\
\text { - } & \text { trômpà } \\
\text { - } & \text { àmîbà }
\end{array}
$$

Presque tous appartiennent au genre $1 / 4$, à l'exception de bìlààdì (8/8), dìspansêrà $(5 / 6)$, kànsêrà $(12 / 13)$

\subsection{Les syntagmes nominaux}

\subsubsection{Déterminé + déterminant}

- dìsaamà dyà mwoyi / dìsaamà dyà mwoyi dyà mucìma

_ dìsaamà dyà manungu 
- dìsaamà dyà mênu

- dìsaamà dyà munda

- dìsaamà dyà tulù

- dìsaamà dyà dicì / dìsaamà dyà macì

- dìsaamà dyà diboba

- dìsaamà dyà mashi / dìsaamà dyà mashi àjika

- dibùùkà dyà mashi

— ditùùlà dyà difù

- dijìmina dyà lungènyì

- dilamuka dyà kamònyi

- dyela dyà munda

- dipweka dyà kalisyumù / dipweka dyà kàlisyumù

- lukosù lwà cyâdi

- lukosù lwà mfwènka

- lwendu lwà mashi

- lukosù lwà mpùta

- lukosù lwà ngondo ìsambòmbò

- musàndà wà bìlààdì / misàndà yà bìlààdì

- musàndà wà ankìlilà / misàndà yà ankililà

- musàndà wà àmîbà / misàndà àmîbà

- mêsù àà mpotà

— bulààlù bwà cileledi

- bwanga bwà misàndà

- kapyà kàà bakìshi

- nzùbu wa bavyeèlà

- nzùbu wa cyondopelu

- nzùbu wa dipandila

— $\quad$ mukàjì nè difù

Type 4: $\mathrm{SN}=\mathrm{N}+\mathrm{SCon}$

Avec un Inf:

$\mathrm{SCon}=$ connecteur $+\mathrm{SV}$

$\mathrm{SV}=\mathrm{V}_{\text {inf }}+\mathrm{N}$

- bwanga bwà kushipa kîshi / twîshi

Avec un $\mathrm{PaV}$

- lukosù lwà lùpàcisha cyâdi

Type 5: SN = N + SLoc

* SCon $=$ Con + Loc

- lumònu lwà pabwîpì 


$$
\begin{array}{ll}
{ }^{*} \text { SLoc } & =\text { Loc }+\mathrm{N} \\
- & \text { mashi mu manungu } \\
- & \text { twîshi mu mênyi } \\
- & \text { mpùta ku bisùlusulu } \\
- & \text { bîshi kumênu } \\
- & \text { twîshi mu bongù } \\
- & \text { mâyi ku bisùlusulu } \\
- & \text { cyuwùjà mu difù }
\end{array}
$$

Type 6: SN = Loc+ Dét

${ }^{*} \mathrm{Loc}+\mathrm{N}$

- ku bapanda

- ku bwanga

- $\quad$ ku balelà

- ku bâna

- ku babùlùka

- kwà mungàngà (mùngàngà)

${ }^{*} \mathrm{Con}+\mathrm{SLoc}+\mathrm{N}$

- byà pa muminu

${ }^{*} \mathrm{Loc}+\mathrm{Con}+\mathrm{SN}$

$\mathrm{SN}=\mathrm{Con}+\mathrm{N}+\mathrm{PaV}$

- $\quad$ kwinshì kwà difù kùsaama

${ }^{*} \mathrm{~N}+\mathrm{Loc}+$ Adj

- mukàjì kuulu kula

Type 7: $\mathrm{SN}=\mathrm{N}+$ Adj

- mashi makesà

Type 8: $\mathrm{SN}=\mathrm{N}+\mathrm{PaNA}$

- mênu àsaama

- mutù ùsaama

Type 9: $\mathrm{SN}=\mathrm{N}+$ PaNA

- makàsà muwùla

Type 10: $\mathrm{SN}=\mathrm{N}+\mathrm{N}$

- munda mpulumuku

- munda mashi

- mubidi kapyà (luuyà/mashìka)

- kampala dibàkà

- cyuwùjà munda 
2. Les formes verbales

\section{$2.1 \quad$ Les infinitifs}

- kwondapa / dyondapa

- kudìàngidisha

- kuditeetesha

- kufùùlulula

- kulènga

- kuumusha

- kupanda

- kuteeta

- kutùùla

- kudìlùnga

\subsection{Les syntagmes verbaux}

\subsubsection{SV à noyau gérondif}

Type 1: Gérondif (verbal) + N

- dijika mashi

- dipeta shokà

- dipangila lupeepèlè

- dijandula bubèèdì / bubèdì

- dyela munda

- dishipa twîshi

Type 2: Gérondif + SN

${ }^{*} \mathrm{SN}=\mathrm{N}+\mathrm{SP}$

$\mathrm{SP}=\mathrm{Loc}+\mathrm{N}$

_ dipeta shokà ku mutù

Gérondif $+\mathrm{SN}$

$\mathrm{SN}=\mathrm{N}+\mathrm{SCon}$

$\mathrm{SCon}=\mathrm{Con}+\mathrm{SLoc}$

$\mathrm{SLoc}=\mathrm{Loc}+\mathrm{N}$

- dikòka mâyi àà mu mwongu

Type 3: Gérondif + SV

${ }^{*} \mathrm{SV}=\mathrm{V}_{\text {inf }}+\mathrm{Adv}$

- dibènga kumòna kula

\subsection{2. $S V$ à noyau infinitif}

${ }^{*} \operatorname{Inf}+\mathrm{N} / \mathrm{SN}$

- kutùùla difù 
- $\quad$ kwela mâyi

- kubàndisha mubidi

_ kupangila (kwà) mupùùyà (lupeepèlè) / dipangila mupùùyà

- kufwà cipùùà

- kwela cipimu

- $\quad$ kupima mubidi

- kupeta shokà

- $\quad$ kwangata mashi àà ku munu $(\mathrm{SN}=\mathrm{N}+\mathrm{SP}$

$\mathrm{SP}=\mathrm{Con}+\mathrm{Loc}+\mathrm{N})$

${ }^{*}$ Inf + Con $+\mathrm{N}$

- kutàngila nè kaamù

- kumònangana nè mungàngà

* Inf + SAdjoint

SAdjoint $=$ Conj + SV

$\mathrm{SV}=\mathrm{V}_{\text {inf }}+\mathrm{N}$

- kupanda bwà kusabula mwâna

\section{Les énoncés}

* P à base prédicative

- mukùjì musòmba mu trômpà

* P à base PaNA

- $\quad$ lwêpù lukèèpèla mu mubidi

* P à base verbale active

- dicì dìsaamisha mbàngà

*P à $\mathrm{PaV}$

- mutù wèla mikenyi

\section{Tableau synthétique}

\begin{tabular}{|c|c|c|c|c|}
\hline \multirow{4}{*}{1} & \multirow{4}{*}{ Formes nominales } & \multirow{4}{*}{137} & Substantifs & 57 \\
\hline & & & Gérondifs & 11 \\
\hline & & & Emprunts & 11 \\
\hline & & & Syntagmes nominaux & 58 \\
\hline \multirow{2}{*}{2} & \multirow{2}{*}{ Formes verbales } & \multirow{2}{*}{32} & Infinitifs & 10 \\
\hline & & & Syntagmes verbaux & 22 \\
\hline \multirow{3}{*}{3} & \multirow{3}{*}{ Les énoncés } & \multirow{3}{*}{4} & Énoncé à base prédicative & 1 \\
\hline & & & Énoncé à base PaNPa & 1 \\
\hline & & & Énoncé à base $\mathrm{PaV}$ & 2 \\
\hline 4 & Totaux & 173 & & 173 \\
\hline
\end{tabular}




\section{ANNEXE 2: Corpus: les termes français-cilubà}

1. ablation: dyumusha, kuumusha

2. alcool: àlùkolà

3. amibiase: misàndà yà àmîbà / musàndà wà àmîbà

4. anémie: dijika dyà mashi, mashi makesà, dìsaamà dyà mashi, dibùùkà dyà mashi

5. angine: byà pa muminu, cilundù

6. antibiotique: bwanga bwà kushipa (kushebeya) twîshi

7. appendicite: kalwalwalwa, àpàndìsità

8. arthrose: dìsaamà dyà manungu

9. asphyxie: dipangila mupùùyà / mupùùyà mupàta

10. asthme: mfwènka, lukosù lwà mfwènka

11. atteinte rénale: tonyi tupàtà/è

12. ausculter: kuteeta nè kaamù / kutàngila nè kaamù

13. avortement: ditùùla difù, kutùùla difù, kutùùla

14. bactérie: kîshi

15. bilharziose: bìlààdì

16. blennorragie: màsàsà

17. bronchite: lukosù lwà lùpàcisha cyâdi

18. broncho-pneumonie: lukosù lwà mpùta, mpùta ku bisùlusulu

19. cancer: kànsêrà / kànsêlà

20. cardiopathie: dìsaamà dyà mwoyi, mwoyi muwùla, dìsaamà dyà myoyi

21. carie dentaire: bîshi ku mênu, mênu àsaama, dìsaamà dyà mênu

22. cataracte: lusòngà

23. centre de santé: nzùbu wa cyondopelu, dìspensêrà

24. céphalée: mutù, mutù ùsaama, mutù wèla mikenyi

25. césarienne: dipanda / kupanda bwà kusabula mwâna

26. chancre dur: tumbulu

27. chirurgie (à la chirurgie): ku bapanda

28. choc: dijìmija lungènyi, kufwà cipùùkà, kupeta shokà

29. choléra: munda mpulumuku, nkòlela

30. cirrhose: mucìma

31. clinique: kìlìnikà

32. coma: cyoyoyi, dilènga, cyonà, dituuta cyonà, kulènga

33. comprimé: kìninà

34. conjonctivite: mêsù àà mpotà / mêsù àà mpòcyà, àpòlo

35. consultation: kudìtàngidisha, kutàngila, kuteeta, kumònangana nè mungàngà, kudìteetesha

36. consultation prénatale: cipimu

37. convulsion: nkooyi, mpaalù, dyuminyina

38. coqueluche: lukosù lwà ngondu ìsambòmbù

39. crise: krîzà, dikùluka 
40. décollement de la rétine: dilamuka dyà kamònyì

41. diabète: siikrà, dyàbêtà

42. diagnostic: dijandula bubèdì

43. diarrhée: munda, dìsaamà dyà munda, dyela (dyà) munda

44. dispensaire: dispensêrà, ku bwanga, kwà mungàngà

45. docteur: mungàngà, doktêrà, ngàngàbukà

46. dysenterie: munda mashi

47. dysménorrhée: kwinshì kwà difù kùsaama

48. éclampsie: makàsà muwùla

49. éjaculer: kwela mâyi

50. éléphantiasis: buzevu

51. épiglottite: kakèlà

52. épilepsie: ciseki, mpaalù

53. épistaxis: milubàlubà, mashi àpàtuka mu dyûlu

54. examen: diteeta

55. femme enceinte: mukàjì nè difù, mukàjì kuulu kula, mukàjì mujìngàkàne

56. fiche: fiishì

57. fièvre: mubidi kapyà / mubidi luuyà / mubidi mashìka, / kubàndisha mubidi

58. filariose: twîshi twà mu mashi, filêrà

59. gale: mpùsù, cikupi

60. gastrite: cifù

61. grossesse extra-utérine: mukùjì musômba mu trômpà, difù mu trômpà

62. hémarthrose: mashi mu manungu

63. hémorroïde: lusundu

64. hépatite: dìsaamà dyà mucìma, mucìma muwùla

65. hôpital: lùpìtaadì, nzùbu wa cyondopelu, òpìtalà

66. hospitaliser: kubwela mu lùpìtaadì / kubwela mu òpìtalà

67. hypertension: lwendu lwà mashi

68. hypocalcémie: dipweka dyà kàlisyumù mu mashi, lwêpu lukèèpèla mu mubidi

69. hypoglycémie: siikrà, dyàbêta

70. hypotension: lwendu lwà mashi

71. infection urinaire: twîshi twà mu mênyi, mìkrôbà

72. infirmier: mungàngà, ngàngàbukà

73. injecter: kutwà lushìngì

74. intoxication: kudilùnga, mulùngù, kunwà bwanga bupìta, dilèwuka

75. jaunisse, ictère: jòònisà, jòònà

76. kinésithérapie: sìyânsà, kìne

77. kwashiorkor: diboba, nsàdi, kwashì

78. laborantin: mungàngà wa ku làbo

79. laboratoire: làbo

80. laryngite: byà pa muminu 
81. leucémie: kansêrà kàà mu mashi

82. liquide amniotique: mâyi

83. lit gynécologique: bulààlù, bulààù bwà cileledi

84. maladie du sommeil: lùbùngù, dìsaamà dyà tulù

85. malaria: màlèyalèya, twîshì twà mu mashi, màlàriya

86. malaria cérébrale: katombùtombù, bwongù bùnyùnguluka, mukupa, dibùluka

87. malnutrition: diboba, dìsaamà dyà diboba, nsàdi

88. maternité (à la maternité): ku balelà, nzùbù wa bavyèla, màtrèmìte

89. matrice: mààtrisà

90. méningite: menènjiità, twîshi mu bwongù

91. microbe: kîshi

92. myopie: dibènga kumòna kula, lumònu lwà pabwîpi

93. neurologie: ku babùlùka

94. opération chirurgicale: dipanda, dipìta ku keelà

95. opérer: kupanda

96. orgelet: kampala dibàkà

97. otite: dìsaamà dyà dicì (macì), minsonjì / misònji

98. otite mastoïde: dìsaamà dyà dicì (macì), dicì dìsaamisha mbàngà

99. otite purulente: tufina mu macì, minsènsa mu macì / minsònji

100. pédiatrie: ku bâna

101. perforation intestinale: mala matùbùùka

102. perfusion: kwela mâyi

103. photographie: fòòto

104. plaie: mpùta

105. plâtre: cì̀to

106. pleurésie: mâyi ku bisùlusulu

107. pneumonie: mwoyi muwùla

108. poche des eaux: kalòwa

109. poliomyélite: tunèka

110. ponction lombaire: dikòka mâyi àà mu mwongu

111. prélever la température: kwela cipimu

112. presbytie: bupofu

113. prostate: pròstatà

114. réanimation: kufùùlulula

115. respiration artificielle: mbòmbonà

116. rhumatisme: binyòkà, ngàlàbàtìzà

117. rougeole: kàntembela

118. salle d'accouchement: cileledi

119. salle d'opération: nzùbù wa dipandila, cipandidi

120. schistosomiase: misàndà yà bìlàà

121. séringue: lushìngì

122. sida: kashèèlèlèkà, sìda, bùdì bwendà

123. sinusite: sìnìzità 
124. soluté: mâyi

125. spermatozoïde: mâyi àà balùmà, bilùmà

126. stérilisation: dishipa twîshi

127. stéthoscope: kaamù kàà mu macì

128. strongyloïde: misàndà yà ànkìlilà

129. syphilis: kàsèndà

130. tétanos: ntèntànosà

131. toux: lukosù

132. traitement: londàpu, dyondapa

133. traumatisme: dipeta shokà, dipèta ku

134. traumatisme cranien: dipeta shokà ku mutù, dipeta ku ku mutù

135. trompe: trômpà

136. trouble mental: ditomboka, katombùtombù, dipaala

137. tuberculose: cyâdi, lukosù lwà tashi / cyâdi

138. tumeur abdominale: cyuwùjà munda, cyuwùjà mu difù

139. typhoïde: cìfòyîdà

140. ulcère de l'estomac: mpùta mucifù

141. urétrite gonococcique: màsàsà

142. vaccin: cisàlu

143. varicelle: makùbùngù

144. variole: mbàlàngà

145. ver: musàndà

146. vermifuge: bwanga bwà misàndà

147. verminose: musàndà

148. video: vìdèo

149. virus: kîshi

150. zona: kapyà / kadilu kàà bakìshi 